\title{
Validation of the Chinese Version of the Modified Gait Efficacy Scale for Patients Removing llizarov External Fixation Device for Over One Year
}

This article was published in the following Dove Press journal: Patient Preference and Adherence

\author{
An Xia ${ }^{1} *$ \\ Xiuli Zhang ${ }^{2, *}$ \\ Yong $\mathrm{Liu}^{3}$ \\ Wen Luo ${ }^{2}$ \\ Zeying $\mathrm{Yu}^{\prime}$
}

'Orthopedics Department, The 2nd Ward of Orthopedics, Tianjin Hospital, Tianjin, People's Republic of China; ${ }^{2}$ The 2nd Ward of Joint Surgery Department, Tianjin Hospital, Tianjin, People's Republic of China; ${ }^{3}$ Orthopedics Department, Tianjin Hexi Hospital, Tianjin, People's Republic of China

*These authors contributed equally to this work
Correspondence: Wen Luo

The 2nd Ward of Joint Surgery, Tianjin Hospital, 406 Jiefangnan Road, Tianjin 3002 II, People's Republic of China

Tel +86 22-131 16190054

Emailwl1984@yahoo.com
Objective: To validate the Chinese version of the modified Gait Efficacy Scale (C-mGES) in people who have had an Ilizarov external fixation apparatus removed more than 1 year ago.

Methods: (1) Translating and cultural adapting the English version mGES into Chinese. (2) Validation of the C-mGES with the Perceived Efficacy Patient-Physician Interactions Scale (PEPPI-10), Self-Efficacy for Rehabilitation Outcome Scale (SER), Lower Extremities Function Assessment Scale (LEFS), and Pain Self-Efficacy Questionnaire (PSEQ). Instrument measurements included item generation, construct validity, reliability testing, testretest reliability and correlation with other scales. Confirmatory factor analysis (CFA) was applied to determine internal consistency and construct validity. One hundred five persons who had Ilizarov external fixation devices removed more than 1 year ago were investigated. Results: One hundred and two patients were included in this research. Our study showed that the C-mGES has high internal consistency (Cronbach's $\alpha$-coefficient 0.928). CFA confirmed good fit indices for a unidimensional model of the C-mGES. In test-retest reliability, 97 patients were analyzed. The results showed that the substantial kappa coefficient is 0.680 , and the ICC is $0.98(95 \% \mathrm{CI})$.

Conclusion: Our study showed that the Chinese version mGES has a good internal consistency, construct validity and satisfactory criterion-related validity. This scale can assist in the assessment of walking self-efficacy in patients who have had Ilizarov external fixation devices removed for over 1 year.

Keywords: gait, self-efficacy, Ilizarov external fixation

\section{Background}

Different opinions on various treatments have been confirmed in many studies, such as tissue transfer, bone grafting, Ilizarov external fixation, and antibiotic cement. ${ }^{1}$ The Ilizarov method has been described as "a panacea for the poor" and has been widely used in developing counties such as South Africa. ${ }^{2}$ Ilizarov technology is considered a safe and effective treatment and is used in orthopedic surgery to correct deformities by lengthening or reshaping limb bones and treating bone fractures. The main purposes of Ilizarov technology are high quality of life and independence. Mobility is an essential component of independent living for postoperative patients with osteopathic trauma. ${ }^{3}$ In recent years, the Ilizarov frame mainly applies in fracture nonunion and limb lengthening orthotics.

Self-efficacy is a central concept in social cognitive theory, which has been developed by Bandura. ${ }^{4}$ It refers to the person's confidence to accomplish a unique 
behavior in different terms. Traditional views proposed that a person's continuity and efficacy are related to the self-efficacy of particular behaviors. ${ }^{5}$ Self-efficacy has been widely used in different fields, including health education and education of teenagers. ${ }^{6}$ At the same time, an increasing number of researchers have confirmed that perceived ability is closely related to an individual's actual performed ability in terms of behavior. Many researchers have designed scales or questionnaires to assess the level of self-efficacy in different populations, and examples are the general self-efficacy scale (GSES), the orthopedic selfefficacy scale (OSES), and the self-efficacy expectation scale (SES). ${ }^{7-9}$

To determine a person's confidence in walking, stepping, and running, the Gait Efficacy Scale (GES) was developed by researchers. The primary version of the GES was developed according to the principle of selfefficacy in social-cognitive theory. Through clinical practice, researchers designed a modified version of the Gait Efficacy Scale. The modified Gait Efficacy Scale (mGES) consists of 10 items to examine an individual's confidence and ability to safely walk on level surfaces and grass, step over an obstacle, step up and down a curb, ascend and descend stairs (with and without a handrail), and walk a long distance. ${ }^{10}$ The results showed that the original version of the modified Gait Efficacy Scale demonstrated test-retest reliability within a four-week period (ICC $=0.93$, $95 \%$ confidence interval $=0.85,0.97)$. The English version of the mGES across the 10 items has high internal consistency (Cronbach's $\alpha$ coefficient $=0.94$ ). In China, study on the confidence in walking for persons with Ilizarov external fixation devices that had been removed over one year ago is in its infancy, and no scale or questionnaire has been available for measurement of a person's selfefficacy or confidence with regard to walking tasks.

To date, the structural validity, discriminant and convergent validity, and test-retest reliability of the Chinese version of the modified Gait Efficacy Scale (C-mGES) have been thoroughly assessed by participants. Therefore, the aim of this research was to assess the internal consistency, structural validity, test-retest reliability, and correlations of the C-mGES in a clinical sample of people who underwent Ilizarov external fixation and have had the Ilizarov external fixator device removed for over one year.

\section{Availability of Data and Material}

No additional data are available.

\section{Methods}

\section{Design}

This observational study used the cross-sectional survey method and is divided into two stages: (1) translation of the original version of the mGES into Chinese; (2) validation of the C-mGES in people with an Ilizarov external fixation device that had been removed for over one year in September 2019.

\section{Data Collection}

According to the requirements of factor analysis, our survey from September to November 2019 required a random sample of 100 persons with Ilizarov external fixation devices that had been removed for over one year. The participants did not have known literacy problems and/or cognitive impairments.

\section{The Requirements of Sample Size in Factor Analysis}

In this clinical study, the sample size passed the minimum requirements, namely that the ratio between the proportion of the number of patients and items should be $>5: 1$, and the sample size should be nearly 10-times larger than the number of items.

\section{Participants}

Patient records of those who had Ilizarov external fixation devices that were removed over one year ago were obtained from a hospital's orthopedic surgery department to participate in the first stage of this study. The Hospital Research Ethics Committee approved the study protocol, and patients provided informed consent prior to participation. At the beginning of this survey, the researchers informed the patients regarding the investigation aims, rights, and obligations in this study. The investigators used uniform advice language to explain the requirements for the questionnaires, and all scales were provided by the patients themselves. The privacy of the patients was protected during the course of the entire study.

The survey was sent between September and November 2019 to a random sample of 105 patients whose records were obtained from the orthopedic surgery department. Only patients with Ilizarov external fixation devices that were removed more than one year ago and radiographically confirmed bone union were included in this research.

The inclusion criteria were as follows: (1) Patients with removed Ilizarov external fixation device for over one year 
ago and radiographically confirmed fracture union; (2) those who were conscious with the ability to comprehend; (3) those who could walk independently; (4) those with no deformed limbs, and (5) those who provided written informed consent.

The exclusion criteria were as follows: Those with (1) conditions affecting the nervous system, such as mental retardation; and (2) symptoms such as deafness, aphasia, and/or the inability to communicate with others.

According to Chinese law for medical research with humans, the Tianjin Hospital Medical Ethics committee provided approval for this study (TJYY-2017-YLS-031). Previously, the investigators explained the aims, rights, and obligations and gained consent from the respondents before data collecting. The privacy of the participants was protected during the study process, and all questionnaires were completed in 15-20 min. At the end of the first survey, patients were asked whether they were willing to complete the C-mGES after approximately 2 weeks.

\section{Translation of the mGES}

The primary version of the mGES consists of 10 items in English. The items are anchored in the statement "How much confidence do you have that you would be able to safely ..." in different situations. Each item is scored on a $0-10$ scale (from 0 to 10 ), providing a score between 0 and 100, which 100 is the best possible gait self-efficacy. ${ }^{10}$

Each item of the English version mGES was translated into the initial Chinese version by two health providers in the orthopedic department at Tianjin Hospital with the permission of the original author (JS Brach). The initial Chinese version was then translated back to English, and reviewed by another senior research fellow. Based on the preliminary results, the final Chinese version mGES was confirmed after discussion. The final Chinese version scale includes 10 items and assesses the confidence to perform different walk tasks also on a 11-point-scale, with 0 indicates not confident and 10 indicates completely confident.

\section{Other Scales for Validation}

Perceived Efficacy Patient-Physician Interactions Scale (PEPPI-10): The original version of the PEPPI-10 was designed by Maly et al, and it measured the confidence of patients in communicating with their physician or doctor for medical information and health education. ${ }^{11}$ The English version of the PEPPI-10 contains 10 items that demonstrated high internal consistency (Cronbach's $\alpha$ coefficient 0.91 ) and satisfactory structural validity and construct validity in community-dwelling elderly people in the USA. ${ }^{12}$ In 2016, Wen Luo et al translated the English version scale into Chinese and tested it on patients with severe knee osteoarthritis, and satisfactory internal consistency was confirmed (Cronbach's $\alpha$ coefficient 0.91). ${ }^{13}$ The study showed that the PEPPI-10 is closely related to the self-efficacy for exercise scale and osteoporosis self-efficacy scale.

Pain Self-Efficacy Questionnaire (PSEQ): The Pain Self-Efficacy Questionnaire consists of 10 items that assess patient accomplishment of unique behavior while under pain. ${ }^{14}$ A study showed that the original version of the PSEQ had satisfactory validity and reliability when used by patients with pain, functional changes, and disability after pain management. ${ }^{15}$ The original version of the PSEQ has subsequently been translated into different languages and is used throughout the world. ${ }^{16}$ The range of the PSEQ score is between 0 and 6, with each item ranging from 0 ("Not at all confident") to 6 ("Completely confident”). A higher score indicates increased selfefficacy while under pain. The Chinese version of the PSEQ was found to have satisfactory internal consistency (Cronbach's $\alpha$ coefficient 0.94 ) and test-retest reliability (intraclass correlation coefficient 0.796 ) after completion in two weeks by patients with lower back pain. ${ }^{17}$

Self-Efficacy for Rehabilitation Outcome Scale (SER): Waldrop and Lightsey designed the English version, which contains 12 items. ${ }^{18}$ On the basis of Bandura's theory, the scale was designed for patients who underwent knee or hip surgery, and the aim was to assess beliefs on physical rehabilitation behaviors. There are 11-Likert items on this scale, with each item ranging from 0 to 10 , and it can measure the confidence of patients on behavior under different situations, for example, pain and emotional distress. The English version of the SER possesses satisfactory internal consistency (Cronbach's $\alpha$ coefficient: 0.94). The Chinese version of the SER exhibits satisfactory reliability and validity. The Cronbach's $\alpha$ coefficient of the Chinese version of the SER was 0.942 in patients with total knee replacement. ${ }^{19}$

Lower extremities function assessment scale (LEFS): This tool has recently been used in many studies, but there have been few reports from China. The LEFS was developed by Binkley et al in 1999, and was widely used in patients with lower limb skeletal and muscular abnormalities. The LEFS consists of 20 projects. ${ }^{20}$ Items are rated on a 5-point Likert scale ranging from 0 to 4 , providing a score range of the scale from $0-80$. A high 
score indicates better functional status. This scale has been translated into Italian, Canadian, French, Dutch, and other languages. The English-language LEFS was translated into Chinese in 2015, and the results showed that satisfactory reliability and validity were obtained from the Chinese version of the LEFS. ${ }^{21}$ The Cronbach's $\alpha$ coefficient was 0.884 , and the split-half reliability coefficient was 0.838 in patients with knee osteoarthritis.

\section{Statistical Analysis}

In this research, we used the SPSS 19.0 (IBM, 2010) and LISREL 8.7 (Scientific Software International, Lincolnwood, IL, USA) software for statistical analysis. We analyzed missing data and frequencies after the initial collection. Structural validity was assessed by confirmatory factor analysis (CFA) using LISREL 8.70. Distributional properties of the C-mGES were inspected to identify possible floor and ceiling effects and to examine the normality of the total scores. ${ }^{22}$

Whether the items in the C-mGES measure a unidimensional construct was examined in the following way. Given the ordinal nature of the items, robust maximum likelihood estimation with Satorra-Bentler (SB) scaled statistics was used. ${ }^{23}$ In addition to the SB chisquare (SB $\chi^{2}$ ) statistic, where smaller values indicate better fit, the non-normed fit index (NNFI), the comparative fit index (CFI), the standardized root-mean-square residual (SRMR), and the root-mean-square error of approximation (RMSEA) were used to examine the model fit. NNFI and CFI values $\geq 0.95$ and SRMR and RMSEA values $\leq 0.08$ and 0.06 , respectively, were considered indicative of satisfactory model fit. ${ }^{24,25}$

Cronbach's $\alpha$ coefficient was used to evaluate the internal consistency of the C-mGES. Cronbach's $\alpha$ coefficient indicated the average split-half reliability coefficient of all the possible items, which was the most common measurement of validity. ${ }^{26}$ The Cronbach's $\alpha$ coefficient score ranged between 0 and 1 . Research has proved that Cronbach's $\alpha$ coefficient measures the internal consistency of the scale. ${ }^{27}$ A Cronbach's $\alpha$ coefficient of more than 0.7 represents satisfactory internal consistency of the scale. ${ }^{28}$ Test-retest reliability was tested by the Cohen's kappa and intraclass correlation coefficient (ICC). ${ }^{29}$

An ICC $\geq 0.70$ was considered adequate for grouplevel comparisons. ${ }^{28}$ Individual agreement between test and retest scores of the C-mGES was assessed using Bland-Altman analysis. ${ }^{30}$ The following values of
Cohen's kappa were used to evaluate the level of agreement: $^{31}<0,0.0-0.20,0.21-0.40,0.41-0.60,0.61-$ 0.80 and $0.81-1.0$ which represent no agreement, slight agreement, fair agreement, moderate agreement, substantial agreement and perfect agreement, respectively.

Pearson's correlation between the sub-scales and total scale was used to indicate the consistency of the content in the C-mGES. If the mGES score showed a normal distribution, Pearson's correlation analysis was used; otherwise, Spearman correlation analysis was used. $\mathrm{P}<0.05$ indicates statistical significance. In this study, we used two-sided test to determine whether these factors could be correlations. The correlation coefficient was used with values of $0.20-0.39$ weak correlation, $0.40-0.59$ moderate correlation, $0.60-0.79$ strong correlation and $0.80-1.0$ very strong correlation. ${ }^{24}$

\section{Results \\ Pre-Testing}

Five patients who previously wore an Ilizarov external fixation device that had been removed for over 1 year were recruited for preliminary testing of the feasibility and readability of the completed C-mGES. The patients evaluated the specific content of the scale, and participated in discussion with the staff (Table 1). The pretesting results indicated satisfactory readability of the C-mGES by patients, and based on this, we adjusted our formulations of the C-mGES.

\section{Patients}

Of the 105 participants recruited, three patients were excluded because of missing responses, leaving 102 for analysis. The clinical and demographic characteristics of the enrolled patients have been shown in Table 2. The median score of the patients who completed the Chinese version mGES was 52.5.

\section{Results of the Adaptation Phase}

During the process of adapting the GES, the research fellows and health providers encountered unclear grammar and concepts that were influenced by different cultural backgrounds. The research fellows reached a consensus on the most appropriate Chinese terminology to assist people with understanding the measurement (Table S1). The results showed that cultural backgrounds were taken into account in the adaptation to ensure that all persons would understand the items of the scale (Table S1). The 
Table I Pre-Testing Patient Characteristics ( $\mathrm{N}=5)$

\begin{tabular}{|l|l|l|l|l|l|l|}
\hline Age (Year) & Sex & Education & Time After Operation (Months) & mGES & LEFS & BMI \\
\hline 58 & Male & Medium & 14 & 66 & 58 & 21.2 \\
53 & Female & High & 16 & 82 & 55 & 20.6 \\
61 & Female & Medium & 15 & 63 & 52 & 21.6 \\
57 & Male & Medium & 18 & 75 & 42 & 20.2 \\
55 & Male & High & 15 & 73 & 46 & 21.8 \\
\hline
\end{tabular}

Table 2 Sample Characteristics $(\mathrm{N}=102)$

\begin{tabular}{|l|l|}
\hline Characteristics & Mean \pm SD or Number \\
\hline Age, years & $61.23 \pm 7.2$ \\
Sex, male & 63 \\
The time after operation, months & $15.35 \pm 2.4$ \\
Ethnicity, Han nationality & 97 \\
\hline Marital status & \\
$\quad$ Not married & 3 \\
Married & 95 \\
Widowed & 4 \\
\hline Education a & \\
Low & 16 \\
Medium & 72 \\
High & 14 \\
\hline mGES (range, 0-100) & $50.40 \pm 13.6$ \\
PEPPI (range, 0-100) & $73.17 \pm 15.0$ \\
SER (range, 0-120) & $114.14 \pm 5.0$ \\
PSEQ (range, 0-60) & $56.30 \pm 3.9$ \\
LEFS (range, 0-80) & $47.03 \pm 10.4$ \\
\hline
\end{tabular}

Notes: ${ }^{a}$ low = none, primary school, lower-level vocational training, lower-level secondary general education; medium $=$ middle-level vocational training, higher-level secondary general education; high = higher-level vocational training, academic education .

Abbreviations: PEPPI-10, Perceived Efficacy Patient-Physician Interactions Scale; SER, Self-Efficacy for Rehabilitation Outcome Scale; PSEQ, Pain Self-Efficacy Questionnaire; LEFS, lower extremities function assessment scale; mGES, modified Gait Efficacy Scale.

researchers believe that the goal of adapting the Chinese version mGES has been achieved.

\section{Distribution Property}

The Chinese version of the modified Gait Efficacy Scale exhibited non-normal distribution, albeit with small-scale skewness (skewness-0.210, kurtosis-0.705). The highest score of the C-mGES was 80, and the lowest was 18 in the current study (Figure 1).

\section{Structural Validity and Internal Consistency}

CFA confirmed good fit indices for a unidimensional model of the C-mGES (chi-square $=78.93, d f=35, \mathrm{P}$ value for Test of Close Fit $=0.020$, root-mean-square error of approximation $(\mathrm{RMSEA}, 90 \% \mathrm{CI})=0.11(0.079 ; 0.14), \mathrm{SB} \chi^{2}(35)$ $=2.26, \mathrm{NNFI}=0.96, \mathrm{CFI}=0.97, \mathrm{SRMR}=0.051$, with the exception of the root-mean-square error of approximation. Standardized factor loading ranged between 0.66 for item 5 and 0.89 for item 10 (Figure 2). The results showed that the Cronbach's $\alpha$ coefficient of the C-mGES is 0.928 with sufficiently high internal consistency. Thus, our results confirmed that the C-mGES fits a unidimensional model.

\section{Test-Retest Reliability}

In test-retest reliability, 5 patients were excluded because of missing responses, leaving 97 for analysis. With substantial kappa coefficient (0.680) (Table 3$)$ and ICC $(0.98 ; 95 \% \mathrm{CI})$, the test-retest reliability of the C-mGES exceeded the cut-off point for group comparisons. Additional Bland-Altman analysis preliminarily confirmed that the limits of agreement

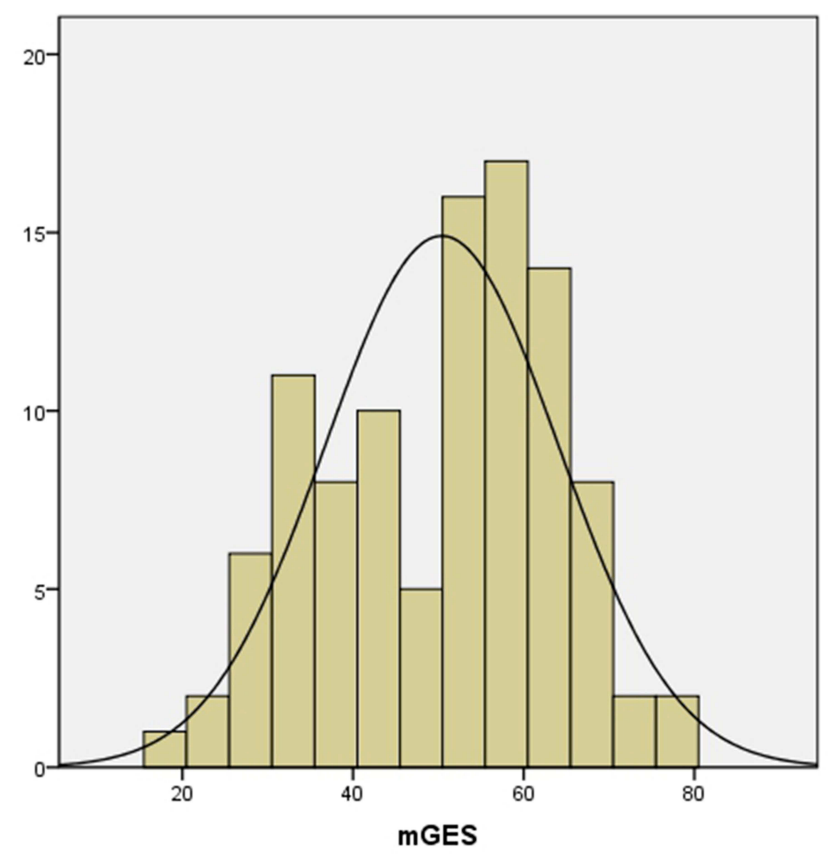

Figure I Patients' mGES score distribution. Mean $=50.4$, Standard deviation (line) $=13.65, \mathrm{~N}=102$.

Abbreviation: $m G E S$, the modified Gait Efficacy Scale. 


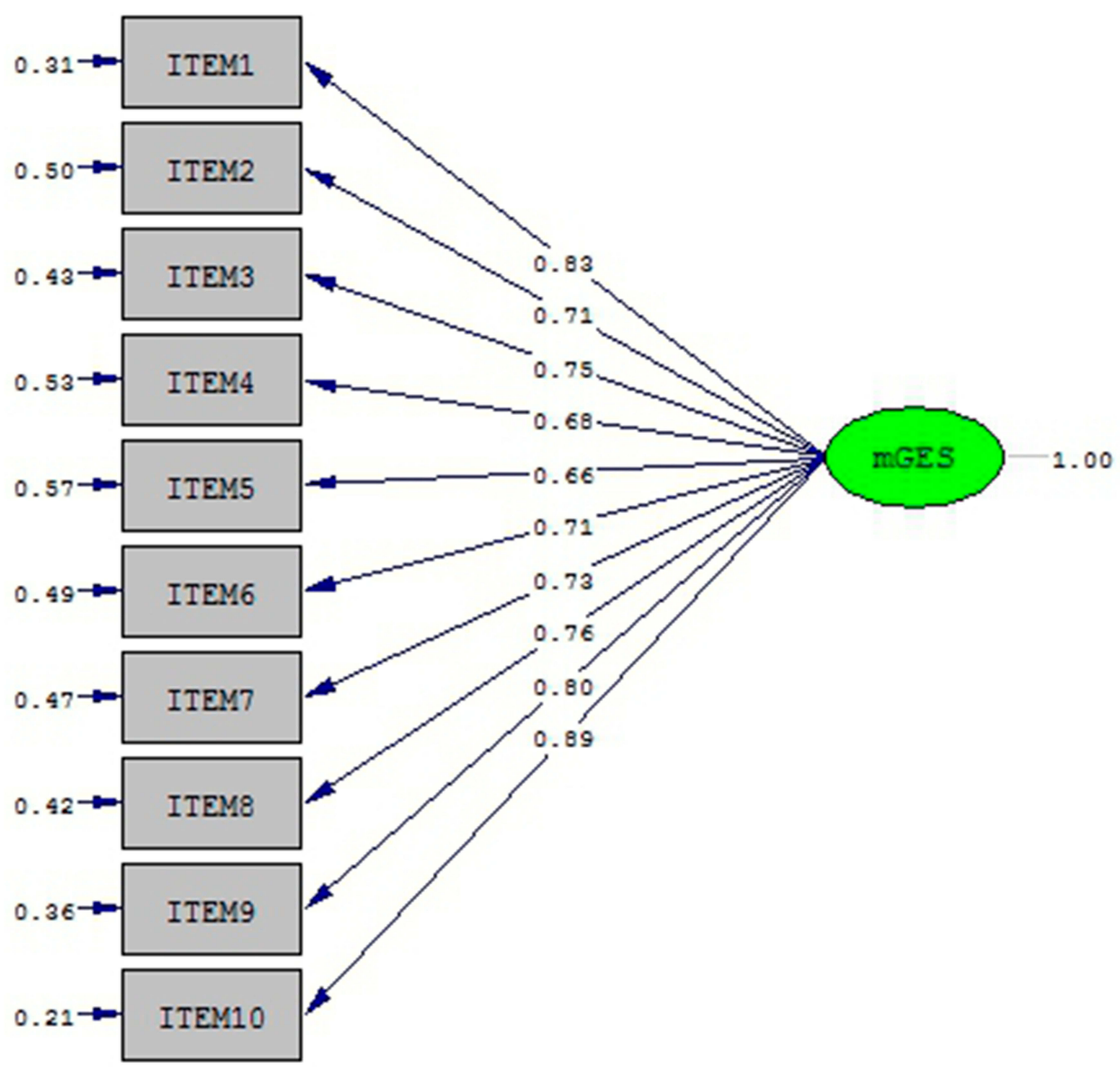

Figure 2 Standardized factor loading and residuals for the items of the mGES. Abbreviation: mGES, the modified Gait Efficacy Scale.

between the two time points were relatively narrow, ranging from -5.7 to 4.1 (Figure 3 ).

\section{Correlation}

The results of Spearman correlation analysis confirmed that the C-mGES moderately correlated with other selfefficacy scales and lower extremities function scales. The results confirmed that the C-mGES exhibited a weak but significant correlation with LEFS $(\mathrm{r}=0.288, \mathrm{P}<0.01)$, which had a moderate but significant correlation with PEPPI-10 $(\mathrm{r}=0.507, \mathrm{P}<0.01)$. In addition, we found that the C-mGES had no correlation with the PESQ ( $\mathrm{r}=-0.141$, $\mathrm{P}>0.05)$ or SER $(\mathrm{r}=0.071, \mathrm{P}>0.05)$ (Table 4).

\section{Discussion}

Our research assesses the validity and reliability of the $\mathrm{C}$-mGES in specific clinical samples. The findings of our study demonstrated that the C-mGES is valid and reliable for patients with removed Ilizarov external fixation devices for over 1 year to examine their walking ability and for interacting with physicians. The C-mGES can be used as an assessment to test the ability to walk in patients who have had their Ilizarov external fixation devices removed for over 1 year, because the scale is simple and valid.

The CFA results showed that the C-mGES is a unidimensional construct. It is a powerful

Table 3 Symmetry Measure of the Chinese Version mGES

\begin{tabular}{|l|l|l|l|l|l|}
\hline & & Value & Asymptotic Standard Error ${ }^{\mathbf{a}}$ & ${\text { Approximation } \mathbf{T}^{\mathbf{b}}}^{\mathbf{P}^{\mathbf{b}}}$ \\
\hline $\begin{array}{l}\text { Coherence measure } \\
\text { Effective cases }\end{array}$ & $\begin{array}{l}\text { Kappa } \\
\mathrm{N}\end{array}$ & $\begin{array}{l}0.680 \\
97\end{array}$ & 0.048 & 36.443 & 0.000 \\
\hline
\end{tabular}

Notes: ${ }^{a} \mathrm{No}$ assumption of zero; ${ }^{\mathrm{b}}$ Assuming zero hypothesis using asymptotic standard error. 


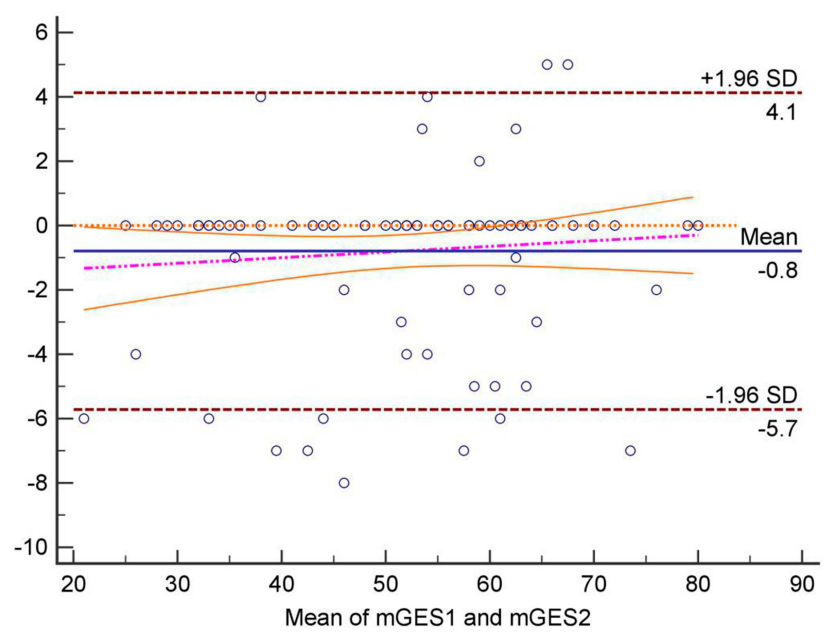

Figure 3 Individual agreement between test and retest scores of the Chinese version $m G E S$. The horizontal solid line represents the mean difference between both scores. The dashed line represents the linear regression line through the data points. The outer dashed lines represent the $95 \%$ limits of agreement (mean difference \pm 1.96 SD of the difference).

measurement in structural validity, and the results demonstrated that the model of the C-mGES fully fits the clinical sample data. Last but not least, previous studies showed that few degrees of freedom of RMSEA values can be inflated in the sample model. ${ }^{25}$ In addition, the C-mGES exhibits high internal consistency, the Cronbach's $\alpha$ coefficient is the same as that reported in the original validation study.

It is important that satisfactory test-retest reliability can discriminate between both scores in the sample. ${ }^{25}$ Based on the test-retest reliability of the Chinese version mGES was above 0.70, which is considered to be a scale that is sufficiently reliable for group comparisons. The kappa coefficient of the C-mGES was 0.680 , which indicates a substantial agreement between the measurements. The additional Bland-Altman analysis indicated that the predominant error was not significant.
Furthermore, the interval time for test-retest reliability and agreement is two weeks in this research. The period is short to ensure that no inter-individual variation occurred.

The results of the correlations between the C-mGES and other scales support the discriminant validity and convergency of the C-mGES. The causes may be that the SER is mainly used for assessing the rehabilitative ability in patients after total hip/knee replacement, and its applied range is circumscribed. However, the C-mGES was significantly correlated with LEFS and PEPPI. Landis et $\mathrm{al}^{31}$ determined that general self-efficacy influences expectations in new situations, while others argued that specific self-efficacy is not assessed by the General Self-Efficacy Scale. ${ }^{32}$ Thus, the C-mGES will be assessed using more domain-specific measures of walking ability.

The study has some limitations. First, the number of patients involved in this study is limited, which likely influenced the comprehensiveness and relevance of this research. Second, owing to the lack of funding for this research, we were unable to perform a Rasch analysis. Despite the above limitations, the results of this research are well supportive of the validity of the C-mGES in a sample of people. The $\mathrm{C}$-mGES is well targeted, and hence, the requirement of a large sample size is reduced. In the current study, we showed the validity and reliability of the C-mGES. Based on these causes, further evidence will be required before the C-mGES can be used for other patients, for example, in those after total knee replacement.

\section{Conclusion}

Our research showed that the Chinese version mGES has satisfactory construct validity and internal consistency. This scale can assist in the assessment of walking selfefficacy in patients who were fitted with Ilizarov external fixation devices and had them removed over one year ago.

Table 4 Spearman Correlations Between mGES and Other Measures

\begin{tabular}{|c|c|c|c|c|c|c|c|}
\hline & & & & LEFS & PSRQ & SER & PEPPI \\
\hline \multirow[t]{7}{*}{ mGES } & \multirow{3}{*}{\multicolumn{3}{|c|}{$\begin{array}{l}\text { Spearman correlations } \\
\text { Sig } \\
\mathrm{N}\end{array}$}} & $0.288^{* *}$ & -0.141 & 0.071 & $0.507^{* *}$ \\
\hline & & & & 0.003 & 0.158 & 0.478 & 0.000 \\
\hline & & & & 102 & 102 & 102 & 102 \\
\hline & \multirow[t]{4}{*}{ Bootstrap $^{a}$} & \multirow{2}{*}{\multicolumn{2}{|c|}{$\begin{array}{l}\text { Deviation } \\
\text { Standard error }\end{array}$}} & 0.007 & -0.002 & 0.000 & -0.007 \\
\hline & & & & 0.109 & 0.103 & 0.099 & 0.097 \\
\hline & & \multirow[t]{2}{*}{$95 \% \mathrm{Cl}$} & Floor & 0.087 & -0.346 & -0.112 & 0.306 \\
\hline & & & Ceiling & 0.502 & 0.069 & 0.279 & 0.685 \\
\hline
\end{tabular}

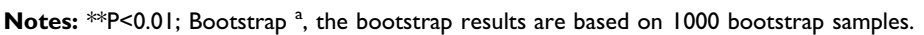


There will be required to provide evidence for psychometric properties in more researches.

\section{Disclosure}

The authors report no conflicts of interest in this work.

\section{References}

1. Ganji SME, Bahrami M, Joukar F. Ilizarov versus AO external fixator for the treatment of tibia open fractures. Iran Red Crescent Med J. 2011;13(12):868-887.

2. Burnei G, Vlad C, Gavriliu S, et al. Upper and lower limb length equalization: diagnosis, limb lengthening and curtailment, epiphysiodesis. Rom J Intern Med. 2012;50:43-59.

3. Foster PA, Barton SB, Jones SC, et al. The treatment of complex tibial shaft fractures by the Ilizarov method. J Bone Joint Surg Br. 2012;94(12):1678-1683. doi:10.1302/0301-620X.94B12.29266

4. Bandura A. Self-efficacy: toward a unifying theory of behavior change. Psychol Rev. 1977;84(2):191-215. doi:10.1037/0033295X.84.2.191

5. Lev EL. Bandura's theory of self-efficacy: applications to oncology. Sch Inq Nurs Pract. 1997;11:21-37.

6. Bandura A. Cognitive regulators. In: Bandura A, editor. Social Foundations of Thought and Action: A Social Cognitive Theory. Englewood Cliffs, NJ: Prentice-Hall; 1986a:467-480.

7. Schwarzer R. The assessment of optimistic self-belies: comparison of the Chinese, Indonesian, Japanese and Korean versions of the general self-efficacy scale. Psychologia. 1997;40:1-13.

8. Pellino T, Tluczek A, Collins M, et al. Increasing self-efficacy through empowerment: preoperative education for orthopedic patients. Orthop Nurs. 1998;17:48-51. doi:10.1097/00006416199807000-00009

9. Resnick B, Jenkins LS. Testing the reliability and validity of the self-efficacy for exercise scale. Nurs Res. 2000;49(3):154-159. doi:10.1097/00006199-200005000-00007

10. Newell AM, Van Swearingen JM, Hile E, et al. The modified gait efficacy scale: establishing the psychometric properties in older adults. Phys Ther. 2012;92(2):318-328. doi:10.2522/ptj.20110053

11. Maly RC, Frank JC, Marsball GN, et al. Perceived efficacy in patient-physician interactions (PEPPI): validation of an instrument in older persons. $J$ Am Geriatr Soc. 1998;46(7):889-894. doi:10.1111/j.1532-5415.1998.tb02725.x

12. Klooster PMT, Oostveen JCM, Zandbelt LC, et al. Further validation of the 5-item perceived efficacy in patient-physician interactions (PEPPI-5) scale in patients with osteoarthritis. Patient Educ Couns. 2012;87(1):125-130. doi:10.1016/j.pec.2011.07.017

13. Zhao H, Luo W, Maly RC, et al. Validation of the Chinese version 10 -item perceived efficacy in patient-physician interactions scale in patients with osteoarthritis. Patient Prefer Adherence. 2016;10: 2189-2195. doi:10.2147/PPA.S110883

14. Nicholas MK. The pain self-efficacy questionnaire: taking pain into account. Eur J Pain. 2007;11:153-163. doi:10.1016/j.ejpain.20 05.12 .008
15. Mangels M, Schwarz S, Sohr G, et al. Der Fragebogen zur Erfassung der schmerzspezifischen Selbst-wirksamkeit (FESS): Eine Adaptation des Pain Self-Efficacy Questionnaire fur den deutschen Sprachraum. Diagnostica. 2009;55:84-93. doi:10.1026/0012-1924.55.2.84

16. Sardá JJ, Nicholas MK, Pimenta CAM, Asghari A. Biopsychosocial predictors of pain, incapacity and depression in Brazilian chronic pain patients. Revista Dor. 2012;13:111-118.

17. Juan $X$, Wenxiang W, Honglin $T$, et al. Testing the reliability and validity of Chinese version pain self-efficacy questionnaire in a population with chronic low back pain. J Nurs Sci. 2013;28 (3):32-34.

18. Waldrop D, Lightsey OR, Ethington CA, et al. Self-efficacy, optimism, health competence and recovery from orthopedic surgery. J Couns Psychol. 2007;48:233-238. doi:10.1037/0022-0167.48.2.233

19. Wang $\mathrm{HY}, \mathrm{Hu}$ YL, Hu SL, et al. The reliability and validity assessment of the Chinese version rehabilitation self-efficacy scale. Chin J Mod Nurs. 2014;20(3):268-270.

20. Binkley JM, Stratford PW, Lott SA, et al. The Lower Extremity Functional Scale (LEFS): scale development, measurement properties, and clinical application. North American Orthopaedic Rehabilitation Research Network. Phys Ther. 1999;79(4):371-383.

21. Nannan L. Lower limb function scale (LEFS) used in patients with knee osteoarthritis in reliability and validity. Capital institute of physical education. 2015.

22. Terwee CB, Bot SD, de Boer MR, et al. Quality criteria were proposed for measurement properties of health status questionnaires. J Clin Epidemiol. 2007;60:34-42. doi:10.1016/j. jclinepi.2006.03.012

23. Joreskog KG, Sörbom D, Du Toit S, et al. LISREL 8: New Statistical Features. Lincolnwood: Scientific Software International; 2001.

24. Browne MW, Cudeck R. Alternative ways of assessing model fit. In: Bollen KA, Long JS, editors. Testing Structural Equation Models. Newbury Park: Sage Publications; 1993:136-162.

25. Hu LT, Bentler PM. Cut-off criteria for fit indexes in covariance structure analysis: conventional criteria versus new alternatives. Struct Equ Model. 1999;6:1-55. doi:10.1080/10705519909540118

26. Cronbach LJ. Co-efficient alpha and the internal structure of tests. Psychometrika. 1951;16:297-334. doi:10.1007/BF02310555

27. Crotina JM. What is co-efficient alpha? An examination of theory and applications. J Appl Psychol. 1993;78:98-104. doi:10.1037/00219010.78.1.98

28. Lohr KN. Assessing health status and quality-of-life instruments: attributes and review criteria. Qual Life Res. 2002;11:193-205. doi:10.1023/A:1015291021312

29. McGraw KO, Wong SP. Forming inferences about some intraclass correlation coefficients. Psychol Methods. 1996;1:30-46. doi:10.1037/1082-989X.1.1.30

30. Bland JM, Altman DG. Statistical methods for assessing agreement between two methods of clinical measurement. Lancet. 1986;327:307-310. doi:10.1016/S0140-6736(86)90837-8

31. Landis JR, Koch GG. The measurement of observer agreement for categorical data. Biometrics. 1977;33(1):159-174. doi:10.2307/ 2529310

32. Sherer M, Maddox JE, Mercandante B, et al. The self-efficacy scale: construction and validation. Psychol Rep. 1982;51:663-671. doi:10.2466/pr0.1982.51.2.663 


\section{Publish your work in this journal}

Patient Preference and Adherence is an international, peer-reviewed, open access journal that focuses on the growing importance of patient preference and adherence throughout the therapeutic continuum. Patient satisfaction, acceptability, quality of life, compliance, persistence and their role in developing new therapeutic modalities and compounds to optimize clinical outcomes for existing disease

states are major areas of interest for the journal. This journal has been accepted for indexing on PubMed Central. The manuscript management system is completely online and includes a very quick and fair peer-review system, which is all easy to use. Visit http:// www.dovepress.com/testimonials.php to read real quotes from published authors. 